\title{
Reflets
}

Revue ontaroise d'intervention sociale et communautaire

\section{L'Ontario au travail et son programme EXPRESS : une analyse critique}

\section{Mary Ann Jenkins, Adje van de Sande et Jean-Marc Bélanger}

Volume 7, numéro 1, printemps 2001

Santé mentale et les défis de l'an 2001

URI : https://id.erudit.org/iderudit/026338ar

DOI : https://doi.org/10.7202/026338ar

Aller au sommaire du numéro

Éditeur(s)

Reflets : Revue ontaroise d'intervention sociale et communautaire

ISSN

1203-4576 (imprimé)

1712-8498 (numérique)

Découvrir la revue

Citer cet article

Jenkins, M. A., van de Sande, A. \& Bélanger, J.-M. (2001). L’Ontario au travail et son programme EXPRESS : une analyse critique. Reflets, 7(1), 90-112.

https://doi.org/10.7202/026338ar

Tous droits réservés (C) Reflets : Revue ontaroise d'intervention sociale et communautaire, 2001
Ce document est protégé par la loi sur le droit d'auteur. L'utilisation des services d'Érudit (y compris la reproduction) est assujettie à sa politique d'utilisation que vous pouvez consulter en ligne.

https://apropos.erudit.org/fr/usagers/politique-dutilisation/ 


\title{
L'Ontario au travail et son programme EXPRESS : une analyse critique
}

\author{
Mary Ann Jenkins \\ Étudiante, Maîtrise en études canadiennes, \\ Université Carleton, Ottawa \\ Adje van de Sande, \\ École de service social, \\ Université Laurentienne, Sudbury \\ Jean-Marc Bélanger \\ École de service social, \\ Université Laurentienne, Sudbury
}

\section{Introduction}

Le 5 mars 1999, Janet Ecker, ministre des Services sociaux et communautaires de l'Ontario, annonçait le lancement d'un nouveau programme, Expérience, poursuite et reprise des études pour les parents (EXPRESS) ${ }^{1}$, obligeant les parents adolescents bénéficiaires de l'aide sociale à terminer leurs études secondaires et à suivre des cours sur le rôle parental. Ce programme obligatoire, qui fait partie de l'initiative L'Ontario au travail, a pour but de rompre le cycle vicieux de la dépendance de ces parents et de leurs enfants à l'égard de l'aide sociale. La raison d'être et la mise en œuvre du programme suscite de vives inquiétudes qu'il importe d'aborder, notamment pour ce qui est de la pauvreté, de la 
dépression maternelle, de la violence, de l'allaitement, du rôle parental et du soin des enfants. Tous ces thèmes sont reliés de près ou de loin à la santé mentale des parents et de leurs enfants.

Cet article est une critique du programme EXPRESS à partir d'une perspective socialiste féministe. L'élaboration de cet article se base sur de nombreuses sources: articles et livres, documents gouvernementaux, communiqués de presse, études sur la pauvreté et la santé, etc. En outre, nous avons consulté des professionnels œuvrant dans le domaine des politiques sociales. Nous passerons brièvement en revue les résultats de trois études, l'une menée par la Région d'Ottawa-Carleton, les deux autres par la Région de Sudbury. Ces études n'ayant pas été menées à partir d'une perspective socialiste féministe, elles ont négligé certains aspects critiques que nous nous permettons de reprendre et d'analyser d'un point de vue à la fois féministe, socialiste et théorique. Pour débuter l'article nous présentons un aperçu de la théorie socialiste féministe.

\section{D'un point de vue théorique}

"Le programme

EXPRESS, tout comme d'autres politiques sociales, comporte certaines suppositions et attentes à l'égard du rôle de la femme qui sont problématiques."
En règle générale, les programmes d'aide sociale ont souvent avantage à être examinés dans une perspective socialiste féministe. Little (1998) remarque que la structure de l'État-providence est biaisée envers les femmes et que plusieurs de ses politiques sociales ne servent qu'à réaffirmer l'oppression subie par les femmes dans l'ensemble du système. Le programme EXPRESS, tout comme d'autres politiques sociales, comporte certaines suppositions et attentes à l'égard du rôle de la femme qui sont problématiques. Ce programme est un exemple frappant de la façon dont le système d'aide sociale en Ontario structure la vie des femmes afin d'atteindre les objectifs néo-libéraux du gouvernement ontarien. Les féministes socialistes sont de l'avis que l'oppression de la femme résulte de sa relation à la fois avec la production et la reproduction (Mitchell 1971; Hartmann 1981). Selon Hartmann (1981), le revenu familial est perçu comme une façon de résoudre le conflit entre le patriarcat et le capitalisme. Les salaires inférieurs des 
"Le travail de la

femme demeure

invisible et sous-

estimé. Les

responsabilités de la

femme au foyer

renforcent ainsi son

infériorité sur le

marché du travail. » femmes assurent la dépendance économique de ces dernières envers les hommes et fortifient l'idée que l'homme et la femme appartiennent à deux sphères distinctes. Le travail de la femme demeure invisible et sous-estimé. Les responsabilités de la femme au foyer renforcent ainsi son infériorité sur le marché du travail. De cette façon, et selon les revenus familiaux, l'homme peut contrôler le travail de la femme au sein comme à l'extérieur de la famille. Cette situation s'avère impossible pour les mères seules, car elles ne reçoivent pas l'appui d'un homme pour subvenir aux besoins de la famille. À contre-coeur, l'État prend alors la place du soutien de famille en fournissant de l'aide sociale. Comme nous le soulignerons plus loin, non seulement l'aide offerte estelle minimale, mais elle entraîne aussi certaines conditions néfastes.

C'est en examinant l'éthique de la famille, par opposition à l'éthique du travail, qu'il est possible de comprendre la bienveillance des femmes. On entend par éthique de la famille les normes idéologiques qui définissent le rôle de la femme au domicile et au travail, et qui relèguent à cette dernière le soin des membres de la famille (Evans 1995). Explicitement et implicitement, la théorie traditionnelle présume généralement que l'attachement principal de la femme est à son foyer et que cet attachement est déterminé biologiquement (Sydie 1987).

Peu à peu, la politique sociale se fait restructurer de façon à être conforme à la ligne d'action de l'entreprise, qui consiste à empêcher les femmes de dépendre de l'État. Un nouveau «citoyentravailleur sans distinction de sexe» surgit des politiques sociales. Il s'agit là d'une entorse au passé, où le "caractère sacré de la maternité» protégeait les femmes de la main-d'œuvre (Scott 1996). Ce phénomène appuie la thèse selon laquelle la société capitaliste dévalue de plus en plus la maternité (Silva 1996). Le financement public affecté au service social pour alléger le travail des femmes au foyer est jugé superflu et est perçu comme une dépense injustifiée, surtout quand l'économie est à la baisse (Armstrong et Armstrong 1987). Selon Evans (1998), les mères seules sont visées en tant que problème social. Les tâches reliées aux soins des enfants ne sont plus considérées comme importantes; c'est le souci de ne pas épuiser les ressources financières de l'État qui prévaut. 
En examinant l'évolution de la politique sociale par rapport aux mères seules, l'importance de réduire la dépendance à l'égard de l'État se révèle clairement. Evans (1996) identifie trois grandes orientations politiques qui s'appliquent aux mères seules en Ontario. Entre 1920 et 1950, les mères seules étaient principalement perçues comme des mères. À compter des années 1960 jusqu'au milieu des années 1980, les politiques ciblaient la femme à la fois comme mère et comme travailleuse. Dans la troisième période, qui commence en 1988, on mine les droits de la femme en matière d'aide sociale et on se concentre dorénavant sur sa capacité à travailler.

Brodie (1996) souligne dans son livre Women and Canadian Public Policy que l'augmentation de la quantité de recherches axées sur les problématiques hommes-femmes démontre que cette restructuration a eu un impact considérable et complexe sur les deux sexes. Cet impact affecte les femmes de façon disproportionnée, en particulier les jeunes femmes, les ainées, les immigrantes, les femmes de race différente et les ouvrières (Leger et Rebick 1995). Pour la suite de cet article, nous nous pencherons sur l'un des groupes de femmes les plus vulnérables: les jeunes mères seules.

\section{Le programme EXPRESS}

"Les mères seules sont dépeintes comme étant négligentes ou irresponsables parce qu'elles sont enceintes et qu'elles dépendent de l'aide sociale."
En considérant la question des adolescentes enceintes et des mères adolescentes, la société insiste souvent sur le «jeu de la moralité» face à la sexualité des adolescentes. Little (1998) avance que selon la plupart des analyses structurales, l'État, et plus particulièrement l'aide sociale, est rarement (ou pour ainsi dire jamais) neutre envers la femme. Les mères seules sont dépeintes comme étant négligentes ou irresponsables parce qu'elles sont enceintes et qu'elles dépendent de l'aide sociale. Le programme EXPRESS a été conçu pour aborder la question de l'augmentation du nombre de mères adolescentes en Ontario. D'après l'Institut Vanier de la famille, bien que le taux de grossesses adolescentes ait chuté de 53,7\% 
"À l'échelle nationale, le revenu moyen des jeunes parents était de $6840 \$$ en 1996, et $45 \%$ de ces parents vivaient sous le seuil de faible revenu..." par 1000 adolescentes en 1974 à $49 \%$ en 1994, la majorité des jeunes femmes choisissent aujourd'hui de garder leur bébé au lieu de le donner en adoption (Ottawa Citizen 2000).

Les jeunes parents vivent souvent en situation de pauvreté. À l'échelle nationale, le revenu moyen des jeunes parents était de $6840 \$$ en 1996, et $45 \%$ de ces parents vivaient sous le seuil de faible revenu (Conseil canadien de développement social 1998). L'éducation est considérée comme un facteur clé pour permettre à ces mères de s'affranchir de l'aide sociale et de rompre le cycle de la dépendance.

Le programme EXPRESS comprend trois éléments: l'expérience, la poursuite et la reprise des études (Gouvernement de l'Ontario 1999). Par expérience, le gouvernement entend, d'une part, l'occasion d'acquérir des compétences reliées à l'emploi et, d'autre part, la participation des parents aux programmes scolaires coopératifs. La formation des jeunes, l'observation au poste de travail, l'obtention d'un emploi à temps partiel ou d'un emploi d'été, puis la planification nécessaire pour accéder au marché du travail ou aux études postsecondaires sont des facteurs désirables reliés à l'expérience.

Quant à la poursuite et à la reprise des études, elle promeut l'importance de terminer ses études secondaires en obligeant les mères à participer à un programme éducationnel en vue d'obtenir leur diplôme. Elle vise aussi à appuyer les participantes qui éprouvent de la difficulté à maîtriser une langue seconde ou qui ont besoin d'aide en matière d'alphabétisation ou en arithmétique, ou encore qui sont désavantagées à d'autres égards. Enfin, il est question d'identifier et de franchir les obstacles qui s'opposent à l'assiduité à l'école et au succès académique.

Ce programme favorise également la croissance et le développement des enfants en encourageant les parents à être de meilleurs aidants et éducateurs. En outre, il est axé sur les besoins des parents et sur les circonstances de la situation particulière du participant. Il offre également des cours sur le rôle parental, auxquels l'adolescente doit consacrer un minimum de 35 heures. 
"Les adolescents âgés de 16 à 17 ans, qu'ils aient des enfants ou non, ne sont pas admissibles à l'aide sociale; ils sont considérés comme enfants et on s'attend à ce qu'ils vivent chez leurs parents ou leurs tuteurs, lesquels ont la responsabilité de subvenir à leurs besoins. "
Les adolescents âgés de 16 à 17 ans, qu'ils aient des enfants ou non, ne sont pas admissibles à l'aide sociale; ils sont considérés comme enfants et on s'attend à ce qu'ils vivent chez leurs parents ou leurs tuteurs, lesquels ont la responsabilité de subvenir à leurs besoins. Toutefois, dans des cas spéciaux, il arrive que certains parents âgés de 16 ou 17 ans aient droit à l'aide sociale. Le programme EXPRESS est obligatoire pour les parents de 16 et de 17 ans qui bénéficient de l'aide sociale et qui n'ont pas terminé leurs études secondaires. Les parents seuls et les couples âgés de 18 à 21 ans qui n'ont pas terminé leurs études secondaires peuvent participer volontairement au programme.

Par l'entremise de L'Ontario au travail, les participants du programme EXPRESS ont accès à une gamme de mesures de soutien qui leur permet de participer à certaines activités reliées au rôle parental, aux études et à l'obtention d'un revenu. Cet appui peut s'offrir soit sous forme de financement pour le transport, les fournitures d'école, les vêtements d'école et les voyages éducationnels, soit sous forme de counseling. Par ailleurs, on verse à tous les participants des subventions pour les services de garde à l'enfance. On s'attend à ce que les communautés se basent sur leurs ressources existantes et qu'elles établissent des liens avec d'autres programmes et services. Tous les programmes sont entrés en vigueur le $1^{\text {er }}$ février 2000 , date qui coïncide avec le début du deuxième semestre dans les écoles secondaires.

Le programme EXPRESS est offert par l'intermédiaire de L'Ontario au travail, une initiative gouvernementale qui oblige les bénéficiaires de l'aide sociale à travailler. Dans le cadre du programme, 25 millions de dollars seront versés annuellement pour des places subventionnées en services de garde d'enfants et pour d'autres services afin d'aider les parents adolescents à se doter de compétences parentales et à terminer leurs études. Les parents qui refusent de participer au programme n'auront pas droit aux prestations de L'Ontario au travail. Bien que la province emploie le terme "parents» par opposition à "mères adolescentes», il n'en demeure pas moins que les bénéficiaires admissibles au programme sont en grande majorité des femmes. 


\section{Aperçu des études}

Dans cette section, nous passons en revue deux études traitant du programme EXPRESS et une étude portant sur le projet Cybermoms à Sudbury. Bien que ces études soient pertinentes en nous donnant de l'information sur ce nouveau programme, elles sont néanmoins limitées en ce qui concerne d'autres aspects plus critiques qui affectent les femmes desservies par le programme EXPRESS. Nous souleverons ces problématiques particulières plus loin dans la partie de l'article sur la discussion.

\section{L'étude menée à Ottawa-Carleton}

Nous examinons d'abord l'étude menée par la municipalité régionale d'Ottawa-Carleton. La ville d'Ottawa se situe dans l'est de l'Ontario; la population de la Région d'Ottawa-Carleton est d'environ 780000 personnes. Perçue pendant longtemps comme une ville gouvernementale somnolente, Ottawa a connu nombre de changements quand l'industrie de haute technologie est devenue l'employeur principal, surpassant la fonction publique. Sur le plan linguistique, $17 \%$ de la population est francophone et $52 \%$ est anglophone (Statistique Canada 1996a). Environ $20 \%$ de la population est immigrante, et $1 \%$ a le statut de Premières nations (Statistique Canada 1996a). De plus, 345 jeunes mères âgées de 15 à 19 ans vivent dans la Région d'Ottawa-Carleton; de ce nombre, $90 \%$ vivent en situation de pauvreté. Elles élèvent souvent leurs enfants seules. Même sans la présence du père de l'enfant, $39 \%$ d'entre elles participent au marché du travail (Statistique Canada 1996b).

Le département de Service social de la Région d'OttawaCarleton a élaboré un projet d'exécution du programme Expérience, poursuite et reprise des études pour les parents (Région d'Ottawa-Carleton 1999) qui se fonde sur les lignes directrices 
"La Région

d'Ottawa-Carleton

accorde présentement un soutien financier à 66 parents adolescents âgés de 16 et de 17 ans, et à 807 parents adolescents de 18 à 21 ans. " provinciales. Le nouveau programme comptera au nombre des projets visant à accroître les mesures de soutien destinées aux jeunes bénéficiaires des prestations de L'Ontario au travail. Le Enhanced Youth Assistance Program (EYAP) fournira un service de gestion de cas, y compris des plans de services, des accords de participation, le maintien de l'admissibilité, le contrôle des cas, la mise en rapports et le financement de mesures de soutien pour les jeunes de 16 et de 17 ans et pour les parents âgés de 18 à 21 ans qui participent à L'Ontario au travail. Le programme EYAP, qui comprend une nouvelle composante reliée à l'administration, offre L'Ontario au travail aux jeunes âgés de 16 à 17 ans. Depuis 1999, l'aide financière n'est affectée qu'à une agence ou à un administrateur au nom des jeunes de 16 à 17 ans. Le message sous-jacent: on ne peut pas se fier sur les jeunes mères pour gérer leurs propres finances.

La Région d'Ottawa-Carleton accorde présentement un soutien financier à 66 parents adolescents âgés de 16 et de 17 ans, et à 807 parents adolescents de 18 à 21 ans. Si un adolescent fait déjà affaire à une agence communautaire et si cette disposition est favorable, on optera pour une administration conjuguée et on lui assignera un coordonnateur de cas du Département de service social.

L'étude d'Ottawa-Carleton (Région d'Ottawa-Carleton 1999) s'est faite par des groupes focus qui rassemblaient 26 jeunes âgées de 16 à 21 ans et qui ont eu lieu au Centre d'Youville, au Bureau des services à la jeunesse et au Centre psycho-social. Ces groupes se composaient en majorité de femmes (88\%), et $84 \%$ des participants avaient la garde de leurs enfants. Les participants ont soulevé divers points importants. Notamment, ils ont souligné que ceux qui désirent obtenir une garde d'enfants autorisée sous licence doivent demeurer sur une liste d'attente pendant un an; qu'en raison d'un manque de flexibilité, les écoles secondaires n'arrivent pas à satisfaire les besoins des parents; que ces derniers préferent faire garder leur enfant dans leur lieu de travail, aspect particulièrement important vu que la plupart des mères allaitent leur enfant; qu'il y a un manque d'information au sujet des programmes et des services, et que les travailleurs sociaux doivent être plus informés au sujet des programmes et des services; que le 
transport pose un problème quand on voyage avec de jeunes enfants (pour se rendre au bureau d'aide sociale, à l'école, à la garderie, aux banques alimentaires, à l'épicerie, etc.); qu'il devrait y avoir plus de visites sur place; et, enfin, qu'il serait avantageux d'avoir accès à un endroit plus propice à la communication privée avec le travailleur social à l'école.

\section{Les études menées à Sudbury}

"À partir du 22 septembre 1999, environ 30 parents âgés de 16 à 17 ans et 180 parents âgés de 18 à 21 ans ont participé au programme L'Ontario au travail dans la Région de Sudbury. "
La ville de Sudbury se situe dans le nord-est de l'Ontario, à environ 400 kilomètres au nord de Toronto. La population de la Région de Sudbury s'élève approximativement à 160000 personnes. Cette ville monoindustrielle se base sur l'exploitation minière. Ces dernières années, son économie s'est diversifiée et elle est maintenant centrée, entre autres, sur les services gouvernementaux, sur l'éducation, sur les soins de santé et sur le tourisme. En outre, $70 \%$ de la population de la Région de Sudbury est anglophone et $30 \%$ est francophone. La ville compte une population immigrante de $8 \%$ et une population autochtone de $3 \%$. Le taux de grossesses adolescentes à Sudbury est au niveau de la moyenne provinciale et se chiffre à 47,1\% par 1000 femmes âgées de 15 à 19 ans. Il importe de noter que le taux de naissances vivantes à Sudbury est de 26,9\%, par rapport au taux provincial de 22,1\% (Kauppi et Picard 1999). Il s'ensuit que le taux d'avortements dans la Région de Sudbury est inférieur à celui de la province (Kauppi et Picard 1999). À partir du 22 septembre 1999, environ 30 parents âgés de 16 à 17 ans et 180 parents âgés de 18 à 21 ans ont participé au programme L'Ontario au travail dans la Région de Sudbury.

L'initiative Les enfants d'abord - donner une chance aux parents rendra accessibles certains services et certaines ressources aux parents et les aidera à franchir les obstacles et à trouver des solutions leur permettant de terminer leurs études secondaires. Pour ce faire, il faut adopter un modèle réunissant les services et les 
ressources dans un même édifice pour que les participants puissent y accéder facilement (Région de Sudbury 2000).

Dans le cadre de la première étude, on a distribué un sondage à 67 personnes (Région de Sudbury 2000) qui fréquentaient 18 écoles différentes; de ce nombre, 13 participants n'étaient pas aux études. Ce sondage a dégagé plusieurs points clés : entre autres, lorsqu'on a demandé aux 67 répondants ce qui constitue un problème de taille, 28 identifient le matériel scolaire, 23 le transport et 14 le soin des enfants. Des quatre réponses possibles dans le sondage, $45 \%$ des répondants ont choisi l'option «autre» pour décrire la personne chargée de la garde des enfants, tandis que $30 \%$ ont indiqué «la famille», $18 \%$ ont opté pour la garderie et $7 \%$ ont déclaré favoriser l'école comme pourvoyeur de services de garderie. Il est intéressant de souligner qu'alors que $21 \%$ d'entre eux ont indiqué que la garde de l'enfant constituait un problème important, la majorité (45\%) des participants ont coché "autre» comme gardien des enfants, ce qui indique que la majorité des parents confiaient la garde de leurs enfants à une garderie sans licence ou à des amis. La Région de Sudbury a organisé des groupes focus, mais les résultats ne sont pas encore disponibles.

La deuxième étude menée à Sudbury portait sur le programme Cybermoms, un projet conçu pour faire de la technologie de l'information un outil qui appuie les adolescentes et les jeunes mères, et qui leur permet de s'assumer pleinement pendant la période prénatale et les 18 premiers mois de la vie de leur enfant. Il importe de souligner que $13 \%$ des mères qui participent à EXPRESS participent également au programme Cybermoms. Ce dernier programme a pour but «d'établir, par l'entremise de la technologie de télécommunications, un réseau de soutien entre les participantes, de leur faciliter l'accès à l'éducation et à la formation, et de leur permettre d'acquérir des aptitudes facilement commercialisables ainsi que des connaissances sur une gamme d'emplois dans le domaine de l'informatique et dans ses champs connexes» (Kauppi et Piccard 1999 : 61). Le revenu moyen des mères adolescentes s'élevait à $675 \$$ par mois, ou à $8100 \$$ par année. En moyenne, les adolescentes versaient $332 \$$ au loyer 
mensuel, ce qui représente approximativement $54 \%$ de leur revenu (Kauppi et Picard 1999).

Les participantes ont été interviewées à différents moments au cours du programme. Lors de ces entrevues, elles ont identifié six grands thèmes:1) l'importance du soutien de la famille; 2) l'appui inadéquat de l'aide sociale;3) l'humiliation et la frustration reliées à l'aide sociale; 4) les stratégies à adopter pour épargner de l'argent; 5) les limites de l'aide sociale; et 6) le manque de soutien de la part du père biologique du bébé.

Ce sont là les principaux résultats des trois études menées dans les régions d'Ottawa et de Sudbury portant sur les jeunes mères adolescentes.

\section{Discussion}

"Des recherches récentes démontrent que les mères se priveront elles-mêmes de nourriture pour s'assurer que leurs enfants aient suffisamment de quoi manger...»
Les trois études que nous avons abordées et les documents consultés ont fait surgir bon nombre de questions. Nous nous attarderons sur six thèmes clés qui ressortent de notre recension des écrits et des trois études citées plus haut. Ces thèmes sont : la pauvreté, la dépression maternelle, la violence, l'allaitement, le rôle parental et le soin des enfants.

D'abord et avant tout, il faut noter la question de la pauvreté. Presque toutes les jeunes femmes qui ont participé aux études vivent en grande pauvreté. Nous savons depuis longtemps que la plupart des familles dépendantes de l'aide sociale n'ont pas un revenu suffisant pour acheter de la nourriture et payer leur loyer (Santé Canada 1994). Des recherches récentes démontrent que les mères se priveront elles-mêmes de nourriture pour s'assurer que leurs enfants aient suffisamment de quoi manger (McIntyre et al. 1998). Un autre problème directement relié à la pauvreté est la question du logement. Les coûts d'hébergement sont si élevés pour plusieurs familles, qu'après avoir payé leur loyer il ne leur reste pratiquement rien pour les vêtements et les fournitures scolaire (Statistique Canada 1996b). De façon générale, dans toute 
"...il faut attendre plusieurs années avant de pouvoir emménager dans un logement subventionné. Dans la Région d'OttawaCarleton la moyenne d'attente est de 5 à 7 ans..." la province de l'Ontario, il faut attendre plusieurs années avant de pouvoir emménager dans un logement subventionné. Dans la Région d'Ottawa-Carleton la moyenne d'attente est de 5 à 7 ans (Child and Youth Health Network of Eastern Ontario 2000). La pauvreté atteint aussi le milieu scolaire et conduit l'enfant à une faible concentration, à un manque de motivation, à un niveau élevé de stress, à un rendement médiocre, à une attitude difficile et à un manque d'assiduité (Association canadienne des commissions scolaires 1997). L'instabilité due à plusieurs déménagements, à l'éviction et au fait d'être sans abri sont aussi des facteurs contribuant à l'abandon scolaire (Ross et Roberts 1999). Ces facteurs, ainsi que l'augmentation de la pauvreté ou la possibilité de tomber en état de pauvreté, affectent la santé mentale des jeunes car ils accroissent leur niveau de stress (Child and Youth Health Network of Eastern Ontario 2000). Les mères seules vivant en état de pauvreté sont donc assujetties, dans leur quotidien, à faire face à des circonstances qui affectent leur santé mentale.

La dépression maternelle est un deuxième thème étroitement lié à celui de la pauvreté. Une étude conduite par Byrne et al. (1998) de l'Université McMaster démontre que $45 \%$ des mères célibataires bénéficiaires de l'aide sociale souffrent de dépression. Dans la population générale, ce taux s'élève à environ $20 \%$. L'une des auteures de ce rapport déclare que l'aide sociale vaut pour très peu pour aider ces mères avec leur santé, surtout lorsqu'il y a absence d'un réseau de santé adéquat. Elle va plus loin en affirmant que la méthode choisie par le gouvernement provincial pour encourager ces femmes à ne plus dépendre de l'aide sociale n'est, en fin de compte, qu'une perte de temps. "On doit cesser de les assommer avec le workfare (travail obligatoire)", dit-elle (Ottawa Citizen 1998 :A5). En 1994, le ministère de la Santé de l'Ontario identifia les enfants des parents souffrant de santé mentale comme un groupe ayant besoin d'aide supplémentaire. Ses recherches démontrent que les enfants dont les parents souffrent de dépression sont plus aptes à avoir des problèmes psychosomatiques que les enfants issues de parents qui ne souffrent pas de dépression (ministère de la Santé de l'Ontario 1994). 
"...si on prenait comme point de départ que le personnel est politique, on pourrait analyser le travail des mères comme faisant partie intégrante de la reproduction sociale. "
La dépression maternelle doit être examinée dans le contexte du «travail des mères» (motherwork). Rosenberg (1987), dans Motherwork, stress, and depression: The costs of privatized social reproduction, examine les origines socio-structurelles possibles de la dépression post-partum. L'explication traditionnelle contemporaine qu'on rattache à la dépression se retrouve dans la fragilité émotionnelle de l'individu. Rosenberg lance un défi à cette façon de penser en offrant une explication basée sur des principes féministes, économiques et politiques. Elle remarque que les conditions sociales rattachées au «travail des mères» sont presque toujours absentes des discussions politiques. On ne discute jamais des effets négatifs telles la dépression, l'anxiété et la violence. Par contre, si on prenait comme point de départ que le personnel est politique, on pourrait analyser le travail des mères comme faisant partie intégrante de la reproduction sociale. Si c'était le cas, être parent serait une forme de main-d'oeuvre sociale. Refuser de reconnaître le travail de mères comme main-d'oeuvre sociale serait inacceptable et se transformerait en une problématique de santé publique.

L'expérience américaine nous donne quelques indices à ce sujet. Les chercheurs du projet Growing Up in Poverty Project (2000) viennent de faire paraitre un rapport intitule Remember the Children: Mothers Balance Work and Child Care UnderWelfare Reform. Ce rapport fait le bilan des deux dernières années sur l'impact du workfare sur les jeunes enfants. Les chercheurs, en provenance des universités de la Californie et de Yale, ont trouvé que le taux de dépression maternelle était trois fois plus élevé chez les mères qui participaient au programme de workfare, que la moyenne nationale. On s'est également rendu compte que certaines pratiques parentales étaient absentes chez ce groupe de femmes, comme par exemple la lecture aux enfants. Ceci est un aspect critique pour le développement de l'enfant qui, en plus de subir l'impact de la dépression maternelle, est aussi affecté par le manque de bonnes pratiques parentales. Pour revenir à cette recherche menée en Californie, $39 \%$ de toutes les femmes interviewées ont exprimé être en accord avec l'énoncé suivant : «À la fin d'une longue journée, je trouve difficile de me sentir chaleureuse et aimable avec mon enfant.» Il devient évident 
que ces deux facteurs - la dépression maternelle et le désengagement parental - peuvent retarder substantiellement le développement du bébé et du jeune enfant. Ceci soulève une question importante en ce qui concerne la promesse de la réforme d'aide sociale qui vise à améliorer l'avancement de l'enfant. Si on n'adresse pas la santé comme une question sociale importante, comment la réforme d'aide sociale contribuera-t-elle, à elle seule, à promouvoir le bien-être des enfants?

La violence est le troisième thème d'importance qui doit être abordé. Une limite des trois études faites dans les régions d'Ottawa et de Sudbury est qu'elles n'ont pas abordé la question de la violence faite aux femmes et aux enfants. En 1993, une étude fut produite par l'organisme Young Mothers' Resource Group, sur les jeunes femmes âgées de 13 à 26 ans de la région de Toronto qui bénéficiaient d'aide sociale. Un tiers des participantes ont rapporté qu'elles étaient maltraitées par leur partenaire actuel. Un tiers d'entre elles avait déjà été maltraitée par leur premier partenaire durant leur grossesse. Il va sans dire que les problèmes psychologiques relatifs à l'estime de soi, à l'anxiété et à l'hypocondrie sont plus prononcés chez les survivantes de viol et d'abus que chez les femmes qui n'ont pas été maltraitées. Une étude canadienne sur la violence faite aux femmes démontre que les jeunes femmes de moins de 24 ans sont quatre fois plus susceptibles d'être maltraitées (Statistique Canada 1993). Ce groupe de femmes

"...il se peut fort bien qu'un bon nombre de femmes ne puissent satisfaire les exigences scolaires requises par le programme EXPRESS parce qu'elles sont en train d'essayer de reconstruire ou de réparer leur vie. " est aussi le plus pauvre de toutes les mères seules. Ces études nous permettent de constater qu'un nombre considérable de femmes qui dépendent de l'aide sociale sont maltraitées ou tentent de mettre fin à des relations caractérisées par le mauvais traitement. Dû à ce genre d'expériences négatives, plusieurs femmes sont également aux prises avec des problèmes émotionnels et de santé mentale. Afin de quitter ces situations abusives, les femmes ont besoin de services d'appui qui sont malheureusement absents ou en nombre insuffisant dans les communautés. À la lumière de ces faits, il se peut fort bien qu'un bon nombre de femmes ne puissent satisfaire les exigences scolaires requises par le programme EXPRESS parce qu'elles sont en train d'essayer de reconstruire ou de réparer leur vie. 


\section{"Le taux}

d'allaitement au sein est moins élevé chez les familles à faible revenu que chez les autres familles, car les femmes qui touchent un maigre revenu sont moins susceptibles de recevoir des renseignements sur l'accouchement et sur l'allaitement maternel.»
Plusieurs des participantes à l'étude menée à Ottawa ont soulevé la question de l'allaitement maternel comme une quatrième thématique importante. Selon l'Organisation mondiale de la santé (OMS/WHO), la méthode optimale pour nourrir son enfant consiste à allaiter exclusivement au sein jusqu'à ce que le bébé atteigne l'âge de 6 mois, puis de continuer l'allaitement maternel, tout en donnant au bébé de la nourriture complémentaire au moins jusqu'à l'âge de 2 ans (WHO 1989;WHA 1994). Le taux d'allaitement au sein est moins élevé chez les familles à faible revenu que chez les autres familles, car les femmes qui touchent un maigre revenu sont moins susceptibles de recevoir des renseignements sur l'accouchement et sur l'allaitement maternel. De plus, les options qu'on leur propose sont souvent présentées dans des messages négatifs ou décourageants (Auerbach 1989). Les femmes retournant sur le marché du travail ou aux études font face à des obstacles laborieux si elles désirent continuer d'allaiter leur bébé. Nous parlons ici de longues heures avec peu de pauses, de pauses trop courtes pour permettre de visiter le bébé ou pour permettre d'extraire le lait à l'aide d'une pompe (un instrument dispendieux), ainsi que d'autres difficultés reliées à l'horaire de la mère et du bébé. Conséquemment, plusieurs mères prennent le chemin de la moindre résistance et choisissent de sevrer le bébé. En général, les mères plus jeunes sont moins disposées que leurs homologues aînées à assumer un rôle maternel adéquat. De plus, leur manque d'éducation et leur dépendance à l'égard de l'aide sociale aggravent le problème et contribuent à leur dévalorisation. Il s'ensuit donc que le cours sur le rôle parental du programme EXPRESS devient une épée à double tranchant. Dans un premier temps, ce programme fournit aux femmes de l'information sur l'allaitement maternel et sur le soin des enfants. Dans un second temps, ce programme les dégage de la responsabilité de rester à la maison comme parents à temps plein pendant la période recommandée par l'Organisation mondiale de la santé, qui s'étale sur une période de deux ans.

Un cinquième thème qui suscite de la controverse avec le programme EXPRESS est l'obligation pour les jeunes mères d'assister à des cours sur le rôle parental. La façon dont les ressources 
"Le programme EXPRESS insiste aussi sur la mise à jour des compétences parentales de la mère. C'est là une attitude prédominante dans notre société,...." sont allouées affecte négativement les femmes quand il est question de demander de l'aide. Les mères âgées de 16 et 17 ans sont stigmatisées car elles sont les seules à devoir suivre des cours sur le rôle parental. Swift (1998) souligne que le courant de pensée dominant actuel met l'accent sur l'immaturité des mères comme cause principale de la négligence de l'enfant. Les recommandations traitant de ce sujet sont donc centrées sur la nécessité de changer l'attitude des mères et d'améliorer leurs compétences parentales. Le programme EXPRESS insiste aussi sur la mise à jour des compétences parentales de la mère. C'est là une attitude prédominante dans notre société, attitude qui fut même reflétée publiquement par le Premier ministre de l'Ontario, Mike Harris, selon lequel la raison pour laquelle les enfants ont faim est "parce que les mères qui travaillent ne cuisent pas de déjeuners chauds pour leurs familles comme elles le faisaient autrefois» (Hurtig 1999). Swift (1998) nous a déjà avisés du fait que sommes tenus de remettre en question la pertinence des services que nous accordons présentement. Il se peut que les cours sur le rôle parental ne réussissent pas à atteindre leurs objectifs s'ils se basent sur le modèle égocentrique de l'individualisme, car il ne fait que perpétuer le blâme sur l'immaturité des mères.

Enfin, la sixième et dernière thématique que nous abordons a été identifiée par les jeunes mères qui ont participé aux études. Il s'agit du soin des enfants. Dans son article The Child-Care Debate: Fading Hopes and Shifting Sands, Ferguson (1998) identifie deux tendances qui ont contribué à miner la légitimité des mères seules qui prennent soin de leurs enfants et qui touchent des prestations d'aide sociale. Comme nous l'avons souligné plus haut, on considère souvent les femmes comme travailleuses, point de vue alimenté par la popularité du travail obligatoire; il s'agit là du deuxième élément du soin compensatoire. Le modèle compensatoire des soins de l'enfant, qui se manifeste dans le programme Bon Départ, propose d'axer les soins sur les enfants venant de milieux défavorisés pour compenser leur manque de ressources et de perspectives d'avenir. Ce modèle compensatoire des soins a été élaboré pour accroittre le potentiel à long terme de l'éducation de l'enfance à risques (Tremblay et Japel 1997). Ceci crée une 
"Certains programmes ont une liste d'attente de plus de deux ans pour ce qui est des places licenciées en services de garde d'enfants (Région d'Ottawa-Carleton 1998). » attitude de deux poids deux mesures à l'égard des mères, attitude déterminée par les classes. Quand les mères dépendent de l'aide sociale, leurs enfants sont perçus comme étant «à risques». Le message que promeut ce modèle, c'est que les mères sont moins en mesure de prendre soin de leurs propres enfants que les garderies. En revanche, quand la mère reçoit un soutien de son partenaire, le modèle traditionnel de la mère à la maison devient préférable pour les enfants (Ferguson 1998).

Les parents qui veulent renoncer à l'aide sociale ont identifié le manque de soins des enfants comme l'obstacle le plus considérable à surmonter (KPMG, cité dans The Child and Youth Network of Eastern Ontario 2000). Certains programmes ont une liste d'attente de plus de deux ans pour ce qui est des places licenciées en services de garde d'enfants (Région d'OttawaCarleton 1998). La situation ne sera pas résolue prochainement, puisque le gouvernement n'a pas approuvé de nouvelles places subventionnées depuis 1993. Même si dans le cadre du programme EXPRESS on a promis 25 millions de dollars pour des places subventionnées en services de garde, les régions d'Ottawa-Carleton et de Sudbury n'ont pas touché à ces fonds. Le programme EXPRESS est quand même en vigueur depuis février 2000, alors qu'attend-t-on pour accroître les services de garde? L'aspect culturel a aussi son importance. Dans la Région d'OttawaCarleton, seulement $11 \%$ des garderies qui détiennent une licence et $8 \%$ des places subventionnées offrent des services en français (Regroupement des services de garde de langue française d'Ottawa-Carleton 1998). Il y a aussi la question du transport qui complique l'accès aux soins des enfants pour les parents qui vivent dans des régions rurales. Il n'est alors pas étonnant que les mères seules aient identifié le soin des enfants comme une priorité.

Malheureusement, des incidents fầcheux se produisent parfois lorsqu'on oblige les gens à se conformer à un système qui ne pourvoit pas tous les systèmes de support nécessaires. Par exemple, on a déterminé que le manque de soins à l'enfant était l'un des facteurs qui a causé la mort d'un enfant de 11 mois en Pennsylvanie aux États-Unis. Sa mère de 17 ans a laissé l'enfant sans supervision dans l'appartement parce qu'elle devait assister à un programme 
"La résolution des problèmes de pauvreté, de violence et de santé est étroitement liée aux besoins des mères seules afin qu'elles puissent se concentrer davantage à développer leurs habiletés parentales. " obligatoire de formation professionnelle. Son fils était malade et la garderie n'avait pas accepté d'en prendre soin ce jour-là. Au lieu de courir le risque de faire suspendre ses prestations si elle n'assistait pas au programme, l'adolescente a laissé son fils à la maison. Malheureusement, la maison a pris feu et l'enfant est mort (Pittsburgh Post Gazette 1999). Cet exemple illustre les conséquences tragiques d'une situation impossible où la mère, prise au piège, a pris une mauvaise décision.

Notre critique du programme EXPRESS a fait ressortir six principaux thèmes clés qui démontrent clairement que, malgré une intention manifeste de venir en aide aux jeunes mères seules, la réussite du programme dépend d'un investissement plus large en termes de ressources afin de bénéficier à l'ensemble de la structure sociale. La résolution des problèmes de pauvreté, de violence et de santé est étroitement liée aux besoins des mères seules afin qu'elles puissent se concentrer davantage à développer leurs habiletés parentales. De plus, si comme société on s'attend à une plus grande implication de leur part sur le marché du travail, il faut aussi développer des services de garde pour enfants appropriés à leurs besoins.

\section{Conclusion}

Au Canada et aux États-Unis, on a de plus en plus tendance à tenir les mères adolescentes pour des boucs émissaires dans la lutte contre la hausse du coût des programmes sociaux. Certains éléments conservateurs de la société canadienne ont réussi à convaincre le public que nous sommes en pleine crise économique. Pour contrer cette crise, on a adopté des politiques sociales qui limitent ou qui éliminent complètement l'accès à l'aide sociale. D'après un sondage gallup effectué en 1994, 86 \% des Canadiens se disent en faveur de contraindre les bénéficiaires de l'aide sociale à travailler. Dans une optique néo-conservatrice, le soin des enfants au sein de la famille est applaudi, et la société est prise de panique morale concernant la destruction de "la famille» (Luxton 1997). 
Le programme EXPRESS, comme bien d'autres prestations et services sociaux, insiste sur la principale responsabilité de la femme, à savoir le soin de sa famille, afin d'assurer qu'elle soit financièrement responsable de ses enfants et d'elle-même. Shragge (1997) critique vigoureusement le fait de mettre l'accent sur l'individu au lieu de mettre l'importance sur les problèmes sociaux et économiques. La plupart des études traitant des politiques sociales se sont attardées à la sphère "publique» ou "politique», et ce, en excluant la sphère "privée» (Little 1998). La situation actuelle que vivent les femmes pauvres et, plus particulièrement, les femmes seules est rarement étudiée ou considérée. Les féministes engagées soutiennent que l'aide sociale se base sur le sexe et que plusieurs de ses politiques contribuent à renforcer tout le système de l'oppression des femmes.

Si l'interdépendance plutôt que l'indépendance devenait la doctrine centrale définissant la citoyenneté, nous serions obligés de reconnaitre le soin des autres comme une responsabilité sociale appuyée par l'État (Baines et al. 1999). Le programme EXPRESS ne reconnait ni la force des ressources personnelles de la femme, ni son niveau de soutien social, ni sa santé mentale. La disponibilité disproportionnée des programmes de soin de l'enfant démontre encore une fois que si l'on met l'accent sur des initiatives manigancées, on passe outre à un élément clé. Au-delà des calculs économiques, ces mères sont assujetties à leurs ressources communautaires (Growing Up in Poverty Project 2000).

Nous devrions continuer d'accorder un soutien aux programmes solides de sécurité au revenu, mais nous devrions également reconnaître qu'en n'abordant pas les inégalités structurales, ils peuvent institutionnaliser la pauvreté de la femme (Brodie 1996). Ces inégalités affectent aussi d'une façon très importante la santé mentale de ces jeunes mères et auront des répercussions à long terme sur leurs enfants. Nos politiques sociales doivent tenir compte du fait que le travail des mères (le motherwork) est un emploi très exigeant et permanent. 


\section{Bibliographie}

ARMSTRONG, P. et H.ARMSTRONG (1987), dans H. Maroney and M. Luxton (sous la dir. de), Feminism and political economy, Toronto, Methuen.

ARMSTRONG, P. et H.ARMSTRONG (1990), Theorizing women's work, Toronto, Garamond Press.

AUERBACH, K. (1989), "The decline of breastfeeding», Mothering , 51, 76-79.

BAINES, C.T., P. EVANS et S. NEYSMITH (1998), Women's caring: Feminist perspective on social welfare, $2^{\text {nd }}$ ed., Toronto, Oxford University Press.

BREASTFEEDING COMMITTEE FOR CANADA (1996), Breastfeeding statement of the Breastfeeding Committee for Canada.

BRODIE, J. (1995), Politics on the margins: Restructuring and the Canadian women's movement, Halifax, Fernwood Publishing.

BRODIE, J. (1996), Women and Canadian Public Policy, Toronto, Harcourt Brace \& Company.

BYRNE, C., G. BROWNE et al. (1998), «Surviving social assistance: 12-month prevalence of depression in sole-support parents receiving social assistance», Canadian Medical Association Journal, $158,881-888$.

CANADIAN COUNCIL ON SOCIAL DEVELOPMENT (1998), The progress of Canada's children, Ottawa.

CANADIAN SCHOOL BOARDS ASSOCIATION (1997), Students in poverty: Towards awareness, action, and wider knowledge, Ottawa.

CHILD ANDYOUTH HEALTH NETWORK OF EASTERN ONTARIO (2000), Thechallenges our children face, Ottawa.

DE SCHWEINITZ, K. (1943), England's road to social security, New York, Barnes.

DOUCET, A. (1995), «Gender equality and gender differences in household work and parenting», Women's Studies International Forum, 18(3), 271-284.

DOUCET, A. (1996), «Encouraging voices: Towards more creative methods for collecting data on gender and household labour», dans L. Morris et S. Lyon (sous la dir. de.), Gender relations in the public and the private, London, Macmillan.

DOUCET, A. (1998), «Interpreting mother-work: Linking methodology, ontology, theory and personal biography», Canadian Woman Studies, 18(2-3), 52-58.

EVANS, P. (1995), «Women and Social Welfare: Exploring the Connections», dans J. Turner et F. Turner (sous la dir. de), Canadian social welfare, Scarborough (Ontario), Allyn and Bacon.

EVANS, P. (1996), «Single Mothers and Ontario's Welfare Policy: Restructuring the Debate», dans J. Brodie (sous la dir. de), Women and Canadian Public Policy, Toronto, Harcourt Brace \& Company.

EVANS, P. (1998), «Gender, Poverty, and Women's Caring», dans C.T. Baines, P. Evans, et S. Neysmith (sous la dir. de), Women's caring: Feminist perspectives on social welfare, Toronto, Oxford University Press.

FER GUSON, E. (1998), «The child-care debate: Fading hopes and shifting sands», dans C.T. Baines, P. Evans et S. Neysmith (sous la dir. de), Women's caring: Feminist perspectives on social welfare, Toronto, Oxford University Press.

GALLUP POLL (1994), 86\% favour making welfare recipients go to work, March 31.

GOVERNMENT OF ONTARIO (1999), Press releases on the LEAP Program, March 5. 
GRAHAM, H. (1983), «Caring: A labour of love», dans Finch et Groves (sous la dir. de), A Labour of Love.

GRAY, B. (1996), Implementation of Ontario Works, Http://leroy.cc.uregina.ca/ rasmussk/resear.html

GROWING UP IN POVERTY PROJECT (2000), Remember the children: Mothers balance work and child care under welfare reform, Berkeley, University of California and Yale University.

GUEST, D. (1985), The emergence of social security in Canada,Vancouver, University of British Columbia Press.

HARTMANN, H. (1981), «The unhappy marriage of Marxism and feminism», dans L. Sargent (sous la dir. de), Women and revolution: A discussion of the unhappy marriage of Marxism and feminism, Boston, South End Press.

HEALTH CANADA (1994), Factors affecting nutritional status of the Canadian population. www.hc.sg.ca/ $\mathrm{hppb} /$ nutrition/pube/cpcs/cpc17.htm

HURTIG, M. (1999), Pay the rent or feed the kids, Toronto, McClelland and Stewart.

KATES, M. (1996), «It's time to talk about birthing without a partner», International Journal of Childbirth Education, 11(1), 26-27.

KAUPPI, C. et L.PICARD (1999), «La pauvreté et les expériences personnelles des mères adolescentes à Sudbury», dans A. van de Sande (sous la dir. de), La pauvreté chez les enfants et les jeunes de Sudbury, Sudbury, Université Laurentienne.

KITCHEN, B. (1995), «Declining living standards in a changing economy», dans J. Turner et F. Turner (sous la dir. de), Canadian social welfare, Scarborough (Ontario), Allyn and Bacon.

LA LECHE LEAGUE (1991), The womanly art of breastfeeding, New York, Plume Books.

LEGER, H. et J.REBICK (1995), The NAC voters' guide, Hull (Québec), National Action Committee on the Status of Women.

LITTLE, M. J. (1998), No car, no radio, no liquor permit:The moral regulation of single mothers in Ontario, 1920-1997, Toronto, Oxford University Press.

LUXTON, M. (1997), «Feminism and families:The challenge off neo-conservatism», dans M. Luxton (sous la dir. de), Feminism and families: Critical policies and changing practices, Halifax, Fernwood.

McINTYER, L. et al. (1998), A glimpse of child hunger in Canada, Ottawa, Applied Research Branch, Strategic Policy, Human Resources Development Canada, October, 27-28.

MITCHELL, J. (1971), Women's estate, New York, Pantheon Books.

MINISTRY OF HEALTH, Premier's Council on Health Well-Being and Social Justice (1994), Ontario Health Survey 1990: Mental health supplement, Toronto, Queen's Printer for Ontario.

MUSTARD, F. (1997), Brain development, competence and coping skills, Toronto, Canadian Institute for Advances Research.

ONTARIO COALITION FOR SOCIAL JUSTICE (1999), Teenaged single mothers and their kids kicked off welfare - if they cannot LEAP, Communiqué de presse, 27 septembre, www.welfarewatch.toronto.on.ca/press/sept27leap.htm

OTTAWA-CARLETON HEALTH DEPARTMENT (1997), Ottawa-Carleton Perinatal Database, Ottawa, Ottawa-Carleton Health Department, Research Unit.

Ottawa Citizen (1998), «Depression rampant among single mothers on welfare», April 7, page A5.

Ottawa Citizen (2000), "Young mothers still waiting for back-to-school allowances», March 16, page A7.

Pittsburgh Post Gazette (1999), www.post-gazette.com/regionstate/19990318fire2.asp 
REGION OF OTTAWA-CARLETON (1999a), Learning, earning and parenting: Implementation plan.

Ottawa, Social Services, September 20.

REGION OF OTTAWA-CARLETON, Health Department (1999b), Starting off well. Ottawa,August.

REGION OF SUDBURY (2000), Children first - Opportunities for parents: Service delivery model, Sudbury (Ontario), Health and Social Services Committee.

REGION OF SUDBURY (1999), Children first - Opportunities for parents: Draftimplementation plan, Sudbury, Health and Social Services Committee.

REGROUPEMENT DES SERVICES DE GARDE DE LANGUE FRANÇAISE D'OTTAWACARLETON (1998), Planification stratégique, Ottawa, avril.

ROSENBERG, H. (1987), «Motherwork, stress, and depression: The costs of privatized social reproduction", dans H. Maroney et M. Luxton (sous la dir. de), Feminism and political economy, Toronto, Methuen.

ROSS. D.P. et P. ROBERTS (1999), Income and child well-being: A new perspective on the poverty debate, Ottawa, Canadian Council on Social Development.

SCOTT, K. (1996), «The dilemma of liberal citizenship:Women and social assistance reform in the 1990's", Studies in Political Economy, 50, summer, 7-36.

SHRAGGE, E. (1997), Workfare: Ideology for a new underclass, Toronto, Garamond Press.

SILVA, E. (1996), «The Transformation of Mothering», dans E. Silva (sous la dir. de), Good enough mothering? Feminist perspectives on lone motherhood, New York, Routledge, 10-36.

STATISTICS CANADA (1993), The Daily, November 18. The violence against women survey highlights, Ottawa, November 18.

STATISTICS CANADA (1996a), 1996 Census: Targeted profile of youth 0-19, Ottawa.

STATISTICS CANADA (1996b), 1996 Census, Zephyr,Vol. 2, No 4.

STATISTICS CANADA (1997), Health Reports,Vol. 9, No 3, Catalogue 82-003-XP8.

SWIFT, K. (1998), «Contradictions in child welfare: Neglect and responsibility», dans C.T. Baines, P. Evans et S. Neysmith (sous la dir. de), Women's caring: Feminist perspectives on social welfare, Toronto, Oxford University Press.

SYDIE, S. (1987), Natural women, cultured men: A feminist perspective on sociological theory, Toronto, Methuen.

TREMBLAY, R. et C. JAPEL (1997), "The long-term impact of quality early child care», Policy Options, 18(1), 7-10.

TURNER, J. (1981), «The Historical Base», dans J. Turner et F. Tunner (sous la dir. de), Canadian social welfare, Toronto, Collier Macmillan Canada, Inc.

VAN DE SANDE, A. (1999), La pauvreté chez les enfants et les jeunes de Sudbury, Sudbury (Ontario), Université Laurentienne.

YOUNG MOTHERS' RESOURCE GROUP (1993), Young mothers in Metro Toronto: Research project, Toronto.

WILENSKY, H. et C. LEBEAUX, C. (1958), Industrial society and social welfare, New York, Russel Sage Foundation.

WORLD HEALTH ASSEMBLY (1994), WHA resolution 47.5 - Infant and young child nutrition, Proceedings $47^{\text {th }}$ World Health Assembly (May 9,1994). 
WORLD HEALTH OR GANIZATION (1989), Protecting, promoting and supporting breastfeeding:The special role of maternity services, Genève.

\section{Note}

1. En anglais, il s'agit du programme Learning, Earning and Parenting (LEAP). 